High Energy Phenomena in Relativistic Outflows III (HEPRO III)

International Journal of Modern Physics: Conference Series

Vol. 8 (2012) 25-30

(C) World Scientific Publishing Company

DOI: $10.1142 / \mathrm{S} 2010194512004370$

\title{
GAMMA RAYS FROM RADIO GALAXIES: FERMI/LAT OBSERVATIONS
}

\author{
PAOLA GRANDI \\ On behalf of the Fermi-LAT collaboration \\ Istituto Nazionale di Astrofisica-IASFBO \\ Via Gobetti 101, I-40129 Bologna, Italy \\ grandi@iasfbo.inaf.it
}

\begin{abstract}
We review the high energy properties of Misaligned AGNs associated with $\gamma$-ray sources detected by Fermi in 24 months of survey. Most of them are nearby emission low power radio galaxies (i.e FRIs) which probably have structured jets. On the contrary, high power radio sources (i.e FRIIs) with GeV emission are rare. The small number of FRIIs does not seem to be related to their higher redshifts. Assuming proportionality between the radio core flux and the $\gamma$-ray flux, several of them are expected to be bright enough to be detected above $100 \mathrm{MeV}$ in spite of their distance. We suggest that beaming/jet structural differences are responsible for the detection rate discrepancy observed between FRIs and FRIIs.
\end{abstract}

Keywords: gamma rays: observations - galaxies: active - galaxies: jets

\section{Introduction}

The Large Area Telescope $\left(\mathrm{LAT}^{1}\right)$ on-board the $\gamma$-ray Fermi satellite detected more than one thousand extragalactic sources ${ }^{2}$ in two years of survey. The majority are blazars [i.e. BL Lacs and Flat Spectrum Radio Quasars (FSRQs)], confirming that AGNs with the jet oriented in the direction of the observer are the brightest $\mathrm{GeV}$ sources. Only a handful of LAT objects have different counterparts, i.e. Starburst galaxies (SBs), Narrow Line Seyfert 1 galaxies (NLSy1s) and Misaligned AGNs $(\mathrm{MAGNs})^{\mathrm{a}}$. The state of the art of extragalactic observations after 24 months of Fermi activity is shown in Fig. 1 where the $\gamma$ spectral slope $\left(\Gamma_{\gamma}\right)$ of blazars and non-blazar objects is plotted as a function of the luminosity $\left(L_{>100 \mathrm{MeV}}\right)$.

In spite of their small number, the non-blazar $\gamma$-ray emitters are extremely appealing, as they offer a powerful physical tool in approaching the high energy phenomena. Starburst Galaxies, for example, are a laboratory for investigating cosmic ray acceleration ${ }^{3}$. The high energy jet emission discovered in NLSY1s is questioning the paradigm according to which radio-loud AGNs are only hosted in elliptical

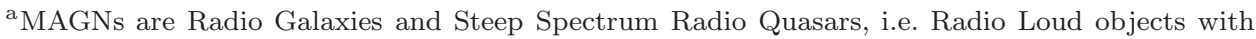
steep radio spectra $(\alpha>0.5)$ and/or showing possibly symmetrical extension in radio maps. 


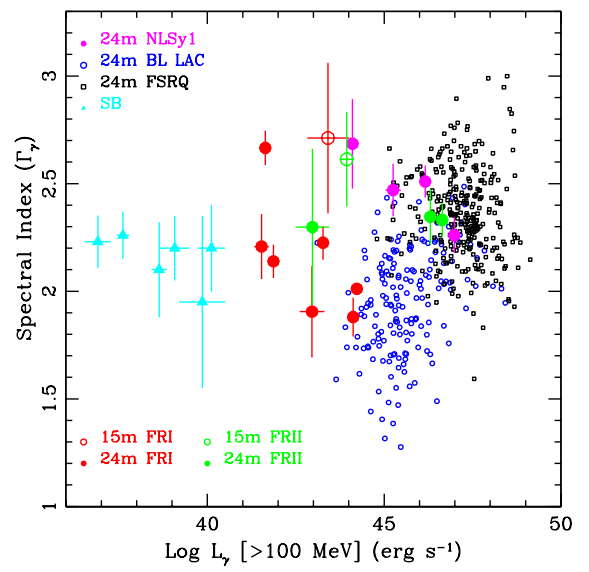

Fig. 1. Spectral slope versus $\gamma$-ray luminosity of the sources detected by the LAT in 24 months. The small group of SBs, NLSy1s and MAGNs preferentially fill the plot region characterized by lower luminosities.

galaxies $^{4}$. The detection of $\gamma$-ray photons in MAGNs is invaluable in revealing the jet structure complexity ${ }^{5}$.

\section{2. $\gamma$-ray properties of Misaligned AGNs}

It is widely accepted that Doppler boosting effects favor the observations of blazars at very high energies. The jet flux amplification $\left(\mathrm{A}=F_{\text {observed }} / F_{\text {intrinsic }}\right)$ is proportional to a power of the kinematic Doppler factor $\delta$ defined as $\delta=[\Gamma(1-\beta \cos \theta)]^{-1}$, being $\beta$ the bulk velocity in units of the speed of light, $\theta$ the angle between the jet and the line of sight and $\Gamma=\left(1-\beta^{2}\right)^{-1 / 2}$ the Lorentz factor. If the observed flux is produced through synchrotron emission, $A=\delta^{3+\alpha}$, where $\alpha$ is the synchrotron power law index. The flux enhancement is strongly dependent on the viewing angle and decreases very rapidly for $\theta>8^{\circ}-10^{\circ}$. For this reason, large inclination Radio Loud AGNs were not considered appealing $\gamma$-ray targets before the Fermi launch. Only some Fanaroff-Riley I and a few Fanaroff-Riley II radio galaxies $(\mathrm{RGs})^{\mathrm{b}}$ were suggested to have fluxes above the LAT sensitivity threshold ${ }^{6-7}$.

The LAT detection of eleven objects ${ }^{5}$ in only 15 months of GeV sky exploration has successively confirmed MAGNs to be a new class of $\gamma$-ray emitters. Among MAGNs, only three source, i.e. 3C111, Centaurus A and NGC6251, have been proposed as candidates by the previous $\gamma$-ray telescope EGRET. The other ones

\footnotetext{
${ }^{\mathrm{b}}$ Following the Fanaroff-Riley ${ }^{8}$ classification, radio galaxies belong to two radio morphological classes corresponding to edge-darkened (FRI) and edge-brightened (FRII) objects, with FRII objects being more powerful $\left(\mathrm{P}_{178 \mathrm{MHz}}>10^{25} \mathrm{~W} \mathrm{~Hz}^{-1} \mathrm{sr}^{-1}\right)$ than FRI radio galaxies. FRIs are considered the parent population of BL Lacs, while FRIIs are thought to be Flat Spectrum Radio Quasars (FSRQs) with jet axis not aligned with the line of sight.
} 
represent a new discovery. Most of the sources of the 15month-MAGN sample are faint $\left(F(>0.1 \mathrm{GeV}) \sim 10^{-8}\right.$ photons $\left.\mathrm{cm}^{-2} \mathrm{~s}^{-1}\right)$ and have steep power law spectra $(\Gamma>2.4)$. This is in general agreement with the AGN Unified Models that assume MAGNs to be a de-boosted version of blazars. Because of their faintness, variability studies are not conclusive. Only in one case, NGC1275, flux and spectral changes could be statistically ascertained on time scale of months ${ }^{9}$. As a consequence, establishing where the $\gamma$-rays are produced is a difficult task. The variability of NGC1275 seems to suggest a sub-pc scale $\left(<10^{18} \mathrm{~cm}\right)$ emission region , but the discovery of $\gamma$-ray emission from the radio lobes of Centaurus $\mathrm{A}^{10}$ shows that extranuclear extended kpc regions can also be sources of high energy photons.

\section{FRII sources: an elusive $\gamma$-ray population}

The 15 month-MAGN sample is dominated by nearby FRI radio galaxies. FRII radio sources appear to be more elusive objects, as also attested by a successive 18 month-study searching for gamma counterparts of Broad Line Radio Galaxies $(\text { mostly showing a FRII radio morphology })^{11}$. In order to investigate this aspect, we take advantage of the publication of the second AGN LAT catalogue $\left(2 \mathrm{LAC}^{2}\right)$ to enlarge the sample of MAGNs. At first the $3 \mathrm{CR}^{12}$, the revised $3 \mathrm{CRR}$ catalog ${ }^{13}$, the Molonglo Southern 4 Jy Sample (MS4) ${ }^{14-15}$ and the $2 \mathrm{Jy}$ sample ${ }^{16}$ were combined (only one entry was considered for the sources listed in more catalogs) to have a large number of radio sources with optical and radio classifications. The demography of the combined sample (3C-MS4-2Jy sample) is represented in Fig. 2 - left panel. Not surprisingly, the FRII is the most crowded class. We intentionally considered the low radio frequency $3 \mathrm{CR} / 3 \mathrm{CRR}$ and MS4 samples because they preferentially select radio sources characterized by steep-spectrum synchrotron emission from extended lobes $^{c}$. Then this big radio sample was cross-correlated with the $2 \mathrm{LAC}$ catalog. The result is shown in Figure 2 -right panel, where the percentages of FRIs, FRIIs, FSRQs, BL Lacs, SBs and AGUs (AGNs with unknown classification) with a $\gamma$-ray association are reported. Some FRI and FRII sources, that are not in the second year catalog (because below the 2LAC adopted TS $>25$ threshold) but were detected on shorter integration (12-18 months) time, are considered in calculating the fraction of detections. BL Lacs and Starbursts have the highest probability to be detected, while FRIs and FSRQs have similar detection rates. Fermi appears to be almost blind to misaligned powerful radio sources. Only $1 \%$ of FRIIs is visible at GeV energies, despite they are the most numerous objects in the 3C-MS4-2Jys combined catalog.

\footnotetext{
${ }^{\mathrm{c}}$ Unlike the $3 \mathrm{CR}, 3 \mathrm{CRR}$ and MS4 catalogs, the $2 \mathrm{Jy}$ sample is a $5 \mathrm{GHz}$ survey able to easily detect bazars. It is useful in comparing MAGN and blazar properties
} 

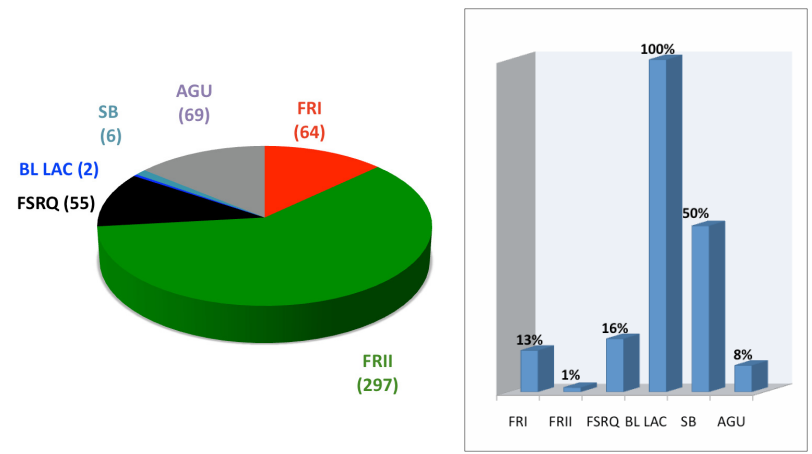

Fig. 2. Left Panel - Number of objects belonging to each class of radio sources. . Right Panel $\gamma$-ray detection rate of each class of radio sources. The percentages of FRIs and FRIIs in the radio source sample are $13 \%$ and $60 \%$, respectively, compared to the detection rate of $1 \%$ and $13 \%$, respectively.

\subsection{Possible interpretations}

The simplest interpretation accounting for the different FRI and FRII detection rates is that we are losing radio powerful MAGNs because more distant (and therefore fainter). In order to verify this possibility, we attempted to estimate the $\gamma$-ray fluxes of the MAGNs for which no GeV association was found. We evaluated the 1 $\mathrm{GeV}$ flux of all the undetected sources, using (in a first approximation) the positive correlation observed between the $5 \mathrm{GHz}$ core fluxes and the $1 \mathrm{GeV}$ flux of the 3C-MS4-2Jy sources with a $\gamma$-ray counterpart (see Fig 3 left panel). We implicitly assumed that $\gamma$-ray emission occurs in similar physical conditions in all AGNs. In agreement with the Spearman test, this correlation has less than a $1 \%$ probability of being due to chance alone and it is still present (chance probability less than $3 \%$ ) when only FRIs and FRIIs are taken into account. Incidentally, we note that a linear gamma-radio relation, considering larger samples of AGNs, has also been reported by other authors ${ }^{17-18}$. In the histogram of Fig. 3-right panel the predicted and observed gamma fluxes for both FRI and FRII sources are compared. Here only the results based on the MAGNs (red line in Fig. 3-left panel) correlations are presented. However a similar histogram was obtained considering the correlation including blazars and AGUs (black line in Fig. 3-left panel). Distance/faintness effects can not explain the FRII scarcity in the $\gamma$-sky. As shown in Fig. 3-right panel, many FRIIs are expected to be as bright as FRIs and above the LAT sensitivity threshold. Different effects must then be invoked to justify the low probability of detecting high powerful radio sources above $100 \mathrm{MeV}$.

It is possible that one of our implicit assumptions, i.e. same flux boosting factor at low and high frequencies in both FRIs and FRIIs, is too simple. For example, the Doppler boosting is stronger and the beaming cone narrower compared to synchrotron processes if the emission is due to Compton scattering of external photons (EC) in the jet ${ }^{19}$. If the high energy emission is dominated (as is likely) 

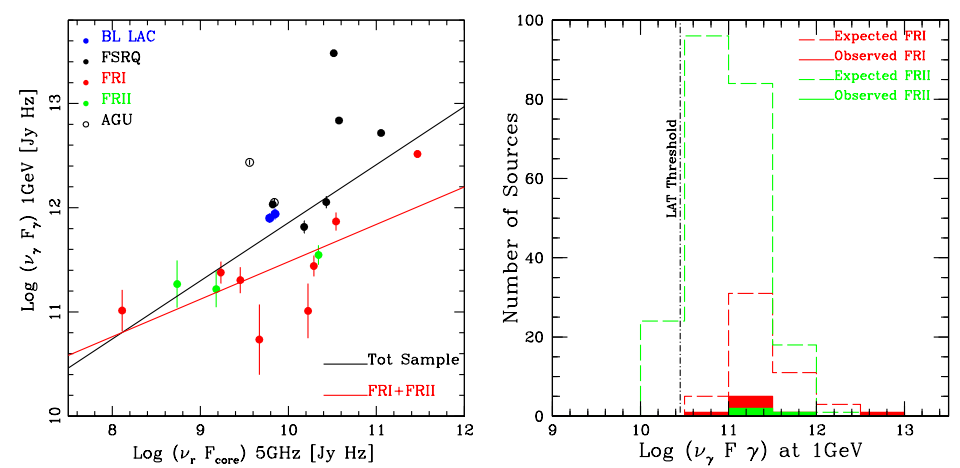

Fig. 3. Left Panel - Radio-Gamma correlation of the 3C-MS4-2Jy objects with a $\gamma$-ray association (black line). A positive (flatter) trend is also present when only FRI and FRI sources are taken into account (red line). Right Panel - Histogram of the predicted fluxes for all the FRI (red) and FRII (green) radio sources belonging to the 3C-MS4-2Jy sample (dashed lines). For comparison, the real observed MAGNs are also shown. Many FRIIs are expected to be bright enough to be detected by Fermi .

by EC processes in powerful radio sources and by SSC processes in low power radio galaxies, a beaming difference could account for the smaller number of FRIIs when compared to FRIs.

It is also probable that one-zone homogeneous SSC/EC models are an oversimplified interpretations of the jet emission ${ }^{20}$. Spectral Energy Distributions (SEDs) of FRI radio galaxies such as NGC1275 ${ }^{21}$, M87 ${ }^{22}$ and $\mathrm{NGC6251^{23 }}$ are consistent with an SSC model with Lorentz factors $(\Gamma \lesssim 3)$, much lower than typical values found in models of BL Lac objects. This is in apparent contrast with the AGN Unification Scheme $^{23}$, unless the assumption of a one-zone homogeneous SSC emitting region is relaxed and a structured jet is assumed (as also attested by recent multifrequency observations and polarization studies ${ }^{24}$ ). Among the proposed scenarios, decelerating jet flow ${ }^{25}$ and spine-layer jet ${ }^{6}$ models seem to be promising in describing the jet complexity ${ }^{23}$. In these models, an efficient (radiative) feedback between different regions increases the IC emission. In one case, the jet is assumed to be decelerating; in the other one, it is supposed to be fast in the inner part and slow in the external envelope. The discrepancy between BL Lac and FRI bulk velocities can then be reconciled. In large inclination RGs we could be observing the external sheets, while in BL Lacs the jetted radiation is directly coming from the inner fast spine. Within this context, the deficit of $\gamma$-ray photons in FRIIs (i.e. in AGNs having powerful accretion disks and poorer hot gas environments ${ }^{\mathrm{d}}$ ) could be due to less prominent (or absent) external layers and/or less efficient deceleration processes. In order to check this possibility, accurate modeling of FRII Spectral Energy Distributions are under investigation.

\footnotetext{
${ }^{\mathrm{d}}$ As well known, the low luminosity FRI sources are on average in richest clusters than high powerful FRII sources ${ }^{26}$
} 


\section{Summary and Conclusions}

Although MAGNs are a minority in the GeV sky, their study is particularly fruitful in providing general insights into the jet structure. We find that more then $10 \%$ of the FRI radio galaxies of the radio sample obtained combining the $3 \mathrm{CR}, 3 \mathrm{CRR}$, MS4 and 2Jy catalogs have $\gamma$-ray associations. Their LAT detections seem to be favored by the presence of different velocity zones in the jet. Only $1 \%$ of FRIIs, belonging to the same 3C-MS4-2Jy sample, are visible above $100 \mathrm{MeV}$. The small number of detections does not seem to be due to their larger distances. More likely, it depends on less favorable jet properties.

\section{Acknowledgments}

This contribution has greatly benefited from the sharing of ideas with C. Dermer, L. Maraschi and G. Ghisellini. P. G. would like to thank G. Palumbo for critical reading of the manuscript, E. Torresi, G. Migliori for stimulating discussions. and V. Bianchin for help with IDL.

\section{References}

1. W. B. Atwood et al., Astrophys. J. 697, 1071 (2009).

2. M. Ackermann et al., submitted to Astrophys. J. (2011). [arXiv:1108.1420].

3. A. A. Abdo et al., Astrophys. J. Lett. 709, L152 (2010).

4. L. Foschini et al. these proceedings.

5. A. A. Abdo et al., Astrophys. J. 729, 912 (2010).

6. G. Ghisellini, F. Tavecchio and M. Chiaberge, Astron. Astrophys. 432, 401 (2005).

7. P. Grandi and G. C. Palumbo, Astrophys. J. 659, 235 (2007).

8. B. L. Fanaroff and J. M. Riley, Mon. Not. R. Astron. Soc. 167, 31 (1974).

9. J. Kataoka et al., Astrophys. J. 715, 554 (2010).

10. A. A. Abdo et al., Science 328, 725 (2010).

11. J. Kataoka et al., Astrophys. J. in press, [arXiv: 1107.3370].

12. H. Spinrad et al. , PASP 97, 932 (1985).

13. R. A. Laing, J. M. Riley and M. S. Longair, Mon. Not. R. Astron. Soc. 204, 1 (1983).

14. A. M. Burgess and R. W. Hunstead, Astron. J. 131, 100 (2006).

15. A. M. Burgess and R. W. Hunstead, Astron. J. 131, 114 (2006).

16. R. Morganti, N. E. B. Killeen and C. N. Tadhunter, Mon. Not. R. Astron. Soc. 263, 1023 (1993).

17. G. Ghirlanda et al., Mon. Not. R. Astron. Soc.,413, 852 (2011).

18. M. Ackermann et al. Astrophys. J., in press. [arXiv:1108.0501].

19. C. D. Dermer, Astrophys. J. 446, L63 (1995).

20. M. M. Reynoso, M. C. Medina and G. E. Romero, Astron. Astrophys. 531, 30 (2011).

21. A. A. Abdo et al., Astrophys. J. Lett. 706, 275 (2009).

22. A. A. Abdo et al., Astrophys. J. 707, 55 (2009).

23. G. Migliori et al., Astron. Astrophys. 533, 72 (2011).

24. J. Gomez, these proceedings.

25. M. Georganopoulos and D. Kazanas, Astrophys. J. 589, L5 (2003).

26. R. M. Prestage and J. A. Peacock, Mon. Not. R. Astron. Soc. 230, 131 (1988). 